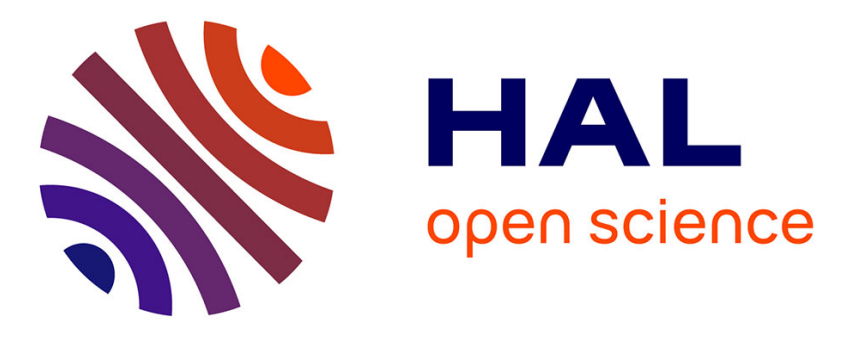

\title{
Inducing Inference Relations from Inconsistency Measures
}

Xiaolong Liu, Philippe Besnard, Sylvie Doutre

\section{To cite this version:}

Xiaolong Liu, Philippe Besnard, Sylvie Doutre. Inducing Inference Relations from Inconsistency Measures. 16th European Conference on Symbolic and Quantitative Approaches to Reasoning with Uncertainty (ECSQARU 2021), Sep 2021, Prague, Czech Republic. pp.486-498, 10.1007/978-3-03086772-0_35. hal-03541660

\section{HAL Id: hal-03541660 https://hal.science/hal-03541660}

Submitted on 25 Jan 2022

HAL is a multi-disciplinary open access archive for the deposit and dissemination of scientific research documents, whether they are published or not. The documents may come from teaching and research institutions in France or abroad, or from public or private research centers.
L'archive ouverte pluridisciplinaire HAL, est destinée au dépôt et à la diffusion de documents scientifiques de niveau recherche, publiés ou non, émanant des établissements d'enseignement et de recherche français ou étrangers, des laboratoires publics ou privés. 


\title{
Inducing Inference Relations from Inconsistency Measures
}

\author{
Xiaolong Liu ${ }^{1,2(\bowtie)}\left(\mathbb{D}\right.$, Philippe Besnard ${ }^{2(凶)}$, and Sylvie Doutre ${ }^{2(\bowtie)}(\mathbb{D}$ \\ 1 Department of Philosophy, Institute of Logic and Cognition, \\ Sun Yat-sen University, Guangzhou, China \\ liuxlong6@mail2.sysu.edu.cn \\ 2 IRIT-CNRS, University of Toulouse, Toulouse, France \\ \{xiaolong.liu,philippe.besnard, sylvie.doutre\}@irit.fr
}

\begin{abstract}
This article defines a family of inference relations which aim at reasoning with inconsistent knowledge bases. These inference relations are defined out of inconsistency measures. We check under which con-ditions the new inference relations satisfy a series of properties for non-monotonic reasoning. We also show that they are paraconsistent when the corresponding inconsistency measures satisfy some rationality postu-lates. Besides, we show some dependencies and incompatibilities among some rationality postulates.
\end{abstract}

Keywords: Inconsistency measures $\cdot$ Inference relations $\cdot$ Rationality

\section{Introduction}

Inconsistency measures are intended to provide a measure of how inconsistent a knowledge base is. Informally speaking, an inconsistency measure is a function which assigns a non-negative real value to a knowledge base with the meaning that larger values indicate a larger inconsistency. As pointed out in Bryson Brown's article [3], an application of inconsistency measures is to induce paraconsistent inference relations, which are able to draw valuable information from inconsistent knowledge bases. Although many inconsistency measures have been proposed so far, there are few inference relations based on them. A drawback of such existing inference relations proposed in [3] is that they are restricted to particular inconsistency measures. It would be desirable to find an approach that works for a broader set of inconsistency measures of the existing literature. To the best of our knowledge, no such general approach exists yet.

It has long been known that a paraconsistent logic, such as LP [11], can be used to define an inconsistency measure $[4,6,10]$. In this article, we investigate the other way around: How to define a paraconsistent inference relation out of a given inconsistency measure. For a discussion on relationships between inconsistency measures and paraconsistent consequence, see [3]. Our idea is to investigate what happens, under an inconsistency measure $\boldsymbol{I}$, when supplementing a knowledge base with the negation of a formula. If $\boldsymbol{I}$ would assign a greater 
inconsistency degree to the knowledge base supplemented with the negation of the formula than to the knowledge base itself, then it would mean somehow that the knowledge base infers the formula. Regarding such an expected behaviour also as a sufficient condition, a notion of inference induced from $\boldsymbol{I}$ appears.

A number of rationality postulates for inconsistency measures have been proposed (e.g., $[5,9,14])$. A survey is provided in [15]. In this article, when an inconsistency measure satisfies some rationality postulates, we will explore which properties are satisfied by the inference relation induced from this measure.

Paper Outline. Section 2 introduces the new inference relations induced from inconsistency measures. Section 3 (resp. Section 4) investigates structural properties (resp. KLM properties) enjoyed by these relations. Section 5 defines paraconsistency and investigates under which conditions the new inference relations are paraconsistent. Section 6 shows dependencies and incompatibility for some rationality postulates. Section 7 concludes and points out future work.

\section{Inference Relations from Inconsistency Measures}

Before introducing the new inference relations, we present some basic notions. We consider a countable set of propositional atoms $\mathcal{A}$. We use $a, b, c, \ldots$ to denote atoms. The resulting logical language, using the connectives $\neg, \vee, \wedge$, and, $\rightarrow$, is denoted $\mathcal{L}$. We use Greek letters, $\varphi, \psi, \ldots$ to denote formulas of $\mathcal{L}$.

We write $\mathcal{K}$ for the set of knowledge bases over $\mathcal{L}$ (where a knowledge base $K$ is a finite set of formulas of $\mathcal{L}$, in symbols: $K \subseteq_{\text {fin }} \mathcal{L}$ ). A minimal inconsistent subset of a knowledge base $K$ is an inconsistent $K^{\prime} \subseteq K$ such that all proper subsets of $K^{\prime}$ are consistent. The set of minimal inconsistent subsets of $K$ is denoted by $\mathrm{MI}(K)$. The set of atoms occurring in $K$ is denoted by $\operatorname{Atoms}(K)$. An atomic injective substitution for $K$ is an injection $\sigma$ : Atoms $(K) \rightarrow \mathcal{A}$. Then $\sigma(\varphi)$ is the formula resulting from $\varphi$ by replacing $a \in \operatorname{Atoms}(K)$ with $\sigma(a)$ simultaneously. We extend $\sigma$ to an injective substitution for $K=\left\{\varphi_{1}, \ldots, \varphi_{m}\right\}$ with $\sigma(K)=\left\{\sigma\left(\varphi_{1}\right), \ldots, \sigma\left(\varphi_{m}\right)\right\}$.

Moreover, we write $\models$ for the consequence relation of classical logic. As we write $\perp$ for falsum, the notation $K \models \perp$ means that $K$ is inconsistent. Logical equivalence is denoted $\equiv$, i.e., $\varphi \equiv \psi$ means that $\varphi \models \psi$ and $\psi \models \varphi$ hold. $K \equiv K^{\prime}$ stands for $K \models \wedge K^{\prime}$ and $K^{\prime} \models \wedge K$. Lastly, a formula $\varphi$ is free for $K$ iff for every consistent subset $K^{\prime}$ of $K, K^{\prime} \cup\{\varphi\} \not \models \perp$. A formula $\varphi$ is safe for $K$ iff $\operatorname{Atoms}(\varphi) \cap \operatorname{Atoms}(K)=\varnothing$ and $\varphi$ is free for $K$.

Definition 1 (Inconsistency measure). A function $\boldsymbol{I}: \mathcal{K} \rightarrow \mathbb{R}_{\geq 0}^{\infty}$ is an inconsistency measure [2] if it satisfies the following two conditions:

Consistency Null: $\boldsymbol{I}(K)=0$ iff $K \not \models \perp$. Variant Equality: If $\sigma$ is an injective substitution then $\boldsymbol{I}(K)=\boldsymbol{I}(\sigma(K))$.

For example, the drastic inconsistency measure $I_{d}$ is a trivial measure that distinguish only between consistent and inconsistent sets of formulas, formally, 
$\boldsymbol{I}_{d}(K)=0$ if $K$ is consistent and 1 if $K$ is inconsistent. $I_{M I}$ counts the number of minimal inconsistent subsets, formally, $\boldsymbol{I}_{M I}(K)=|\mathrm{MI}(K)|$.

We are now in the position to define an inference relation induced from an inconsistency measure, according to the intuition introduced in Sect. 1. That is, we are about to define $K \Vdash \varphi$ as $\boldsymbol{I}(K \cup\{\neg \varphi\})>\boldsymbol{I}(K)$. However, we slightly amend this in the actual definition below because we must take care of the special case $\neg \varphi \in K$ (which obviously gives $\boldsymbol{I}(K \cup\{\neg \varphi\})=\boldsymbol{I}(K)$ no matter what).

Definition 2 (Induced inference relations). Let $\boldsymbol{I}$ be an inconsistency measure. For any $K \in \mathcal{K}$, the inference relation $\Vdash$ induced from $\boldsymbol{I}$ is given by

$$
K \Vdash \varphi \text { iff } \boldsymbol{I}(K \cup\{\neg \varphi\})>\boldsymbol{I}(K \backslash\{\neg \varphi\}) .
$$

Lemma 1. Let $\boldsymbol{I}$ be an inconsistency measure.

$$
K \Vdash \varphi \text { iff } \begin{cases}\boldsymbol{I}(K \cup\{\neg \varphi\})>\boldsymbol{I}(K) & \text { if } \neg \varphi \notin K \\ \boldsymbol{I}(K)>\boldsymbol{I}(K \backslash\{\neg \varphi\}) & \text { if } \neg \varphi \in K\end{cases}
$$

Lemma 2. $K \Vdash \varphi$ iff $K \backslash\{\neg \varphi\} \Vdash \varphi$.

It is desirable that for any consistent knowledge base, $\Vdash$ gives the same consequences as classical logic. The following theorem guarantees this.

Theorem 1. Let $\boldsymbol{I}$ be an inconsistency measure. For all consistent $K \in \mathcal{K}$, $K \Vdash \varphi$ iff $K \models \varphi$.

Proof. Take a consistent knowledge base $K$. $(\Rightarrow)$ Suppose $K \Vdash \varphi$. By Definition 2, $\boldsymbol{I}(K \cup\{\neg \varphi\})>\boldsymbol{I}(K \backslash\{\neg \varphi\})$. It is the case that $\boldsymbol{I}(K \backslash\{\neg \varphi\})=0$, due to Consistency Null and the consistency of $K \backslash\{\neg \varphi\}$. Hence $\boldsymbol{I}(K \cup\{\neg \varphi\})>0$. By Consistency Null, I $(K \cup\{\neg \varphi\})>0$ iff $K \cup\{\neg \varphi\}$ is inconsistent. Therefore, $K \models \varphi$. $(\Leftarrow)$ Suppose $K \models \varphi$. Then $K \cup\{\neg \varphi\}$ is inconsistent. Applying Consistency Null yields $\boldsymbol{I}(K \cup\{\neg \varphi\})>0$ and $\boldsymbol{I}(K \backslash\{\neg \varphi\})=0$. Hence, $\boldsymbol{I}(K \cup\{\neg \varphi\})>\boldsymbol{I}(K \backslash\{\neg \varphi\})$. That is, $K \Vdash \varphi$ by Definition 2 .

Before proceeding, we must pay special attention to a rationality postulate called Free-Formula Independence [2], which can be written in two ways:

Set Union version: If $\varphi$ is free for $K$ then $\boldsymbol{I}(K \cup\{\varphi\})=\boldsymbol{I}(K)$.

Set Difference version: If $\varphi$ is free for $K$ then $\boldsymbol{I}(K)=\boldsymbol{I}(K \backslash\{\varphi\})$.

Lemma 3. The two versions of Free-formula Independence are equivalent.

Proof. The first case is $\varphi \in K$. We start to prove that set union version entails set difference version. Assume that $\varphi$ is free for $K$. Then $\varphi$ is also free for $K \backslash\{\varphi\}$. By set union version, $\boldsymbol{I}((K \backslash\{\varphi\}) \cup\{\varphi\})=\boldsymbol{I}(K \backslash\{\varphi\})$. That is, $\boldsymbol{I}(K)=\boldsymbol{I}(K \backslash\{\varphi\})$ due to the assumption $\varphi \in K$. The converse is obvious as $\boldsymbol{I}(K \cup\{\varphi\})=\boldsymbol{I}(K)$ always holds due to the assumption $\varphi \in K$.

The second case is $\varphi \notin K$. It is obvious that set union version entails set difference version, since $\boldsymbol{I}(K)=\boldsymbol{I}(K \backslash\{\varphi\})$ always holds due to the assumption 
$\varphi \notin K$. To prove the converse, we assume that $\varphi$ is free for $K$. It suffices to show that $\varphi$ is also free for $K \cup\{\varphi\}$. We assume for the contradiction that $\varphi$ is not free for $K \cup\{\varphi\}$. Then there exists a consistent subset $K_{1}$ of $K \cup\{\varphi\}$ such that $K_{1} \cup\{\varphi\} \models \perp$ by the definition of free formulas. If $\varphi \in K_{1}$, then we have $K_{1} \models \perp$ which contradicts the consistency of $K_{1}$. If $\varphi \notin K_{1}$ (which entails $\left.K_{1} \subseteq K\right)$, then the assumption $K_{1} \cup\{\varphi\} \models \perp$ contradicts the assumption that $\varphi$ is free for $K$. To sum up, $\varphi$ is free for $K \cup\{\varphi\}$. Immediately, by set difference version, $\boldsymbol{I}(K \cup\{\varphi\})=\boldsymbol{I}((K \cup\{\varphi\}) \backslash\{\varphi\})$. That is, $\boldsymbol{I}(K \cup\{\varphi\})=\boldsymbol{I}(K)$.

Lemma 4. Let I satisfy Free-formula Independence. The following items hold: (i) If $\neg \varphi$ is free for $K$ then $K \forall \varphi$. (ii) If $\varphi$ is free for $K$ then $K \forall \neg \varphi$.

Proof. (i) If $\neg \varphi \notin K$, apply Free-formula Independence, set union version, to get $\boldsymbol{I}(K \cup\{\neg \varphi\})=\boldsymbol{I}(K)$ and Lemma 1 to obtain $K \forall \varphi$. If $\neg \varphi \in K$, apply the set difference version to get $\boldsymbol{I}(K)=\boldsymbol{I}(K \backslash\{\neg \varphi\})$ and Lemma 1 to obtain $K \Downarrow \varphi$. Then, apply Lemma 3. (ii) Since $\varphi$ is free for $K, \neg \neg \varphi$ is free for $K$. The remaining proof is similar to (i).

\section{$3 \quad$ Structural Properties Checking}

Tarski [13] and Scott [12] worked out the idea of a logic as a closure operator, i.e., an operator over sets of formulas which satisfies the so-called Extension, Idempotence, and Isotony. Since we have formulated the induced consequence as a relation $\Vdash$, they can be expressed, assuming compactness, as follows.

Reflexivity $K \Vdash \varphi$ for any formula $\varphi \in K$.

Monotony If $K \Vdash \varphi$ then $K \cup\{\psi\} \Vdash \varphi$.

Cut If $K \Vdash \varphi$ and $K \cup\{\varphi\} \Vdash \psi$ then $K \Vdash \psi$.

We now investigate the question of which properties of $\boldsymbol{I}$ guarantee $\Vdash$ to satisfy Reflexivity, Cut, and Monotony. ${ }^{1}$ We will use the following postulates of the literature $[1,15]$.

Penalty: For $\varphi \notin K$, if $\varphi$ is not free for $K$ then $\boldsymbol{I}(K \cup\{\varphi\})>\boldsymbol{I}(K)$.

Monotony: If $K \subseteq K^{\prime}$ then $\boldsymbol{I}(K) \leq \boldsymbol{I}\left(K^{\prime}\right)$.

Rewriting: If $\psi$ is a prenormal form ${ }^{2}$ of $\varphi$ then $\boldsymbol{I}(K \cup\{\varphi\})=\boldsymbol{I}(K \cup\{\psi\})$.

Proposition 1. Let $\boldsymbol{I}$ satisfy Penalty. Then, $K \Vdash \varphi$ for all consistent $\varphi \in K$ (i.e., $\Vdash$ satisfies Reflexivity except for inconsistent formulas).

${ }^{1}$ Using Monotony, it is clear that Cut implies the finite general form: If $K \Vdash \varphi_{i}$ for $i=1, \ldots, n$ and $K \cup\left\{\varphi_{1}, \ldots, \varphi_{n}\right\} \Vdash \psi$ then $K \Vdash \psi$. Similarly, Monotony gives the finite general form: If $K \Vdash \varphi$ then $K \cup\left\{\psi_{1}, \ldots, \psi_{n}\right\} \Vdash \varphi$.

${ }^{2} \psi$ is a prenormal form of $\varphi$ if $\psi$ results from $\varphi$ by applying one or more of following principles: commutativity, associativity and distribution for $\wedge$ and $\vee$, De Morgan laws, double negation equivalence. 
Proof. Consider a consistent formula $\varphi$ of $K$. Thus, $\neg \varphi$ is not free for $K$ due to $\{\varphi\}$ being a consistent subset of $K$ such that supplementing it with $\neg \varphi$ gives an inconsistent set: $\{\varphi, \neg \varphi\} \models \perp$. Assume first $\neg \varphi \notin K$. By Penalty, $\boldsymbol{I}(K \cup\{\neg \varphi\})>$ $\boldsymbol{I}(K)$. Then, $K \Vdash \varphi$ ensues by Lemma 1. Assume $\neg \varphi \in K$ instead. Since $\varphi$ is in $K$ and $\varphi \not \forall \perp$, it happens that $\{\varphi\}$ is a consistent subset of $K \backslash\{\neg \varphi\}$ (in view of the trivial fact $\varphi \neq \neg \varphi$ ) such that supplementing it with $\neg \varphi$ gives an inconsistent set. That is, $\neg \varphi$ is not free for $K \backslash\{\neg \varphi\}$. Also, $\neg \varphi$ is not in $K \backslash\{\neg \varphi\}$. Then, Penalty applies to give $\boldsymbol{I}((K \backslash\{\neg \varphi\}) \cup\{\neg \varphi\})>\boldsymbol{I}(K \backslash\{\neg \varphi\})$. By Lemma $1, K \backslash\{\neg \varphi\} \Vdash \varphi$. Applying Lemma $2, K \Vdash \varphi$.

Please observe that for an inconsistent $\varphi$ in $K$, having $K \Vdash \varphi$ would amount to $\boldsymbol{I}(K \cup\{\neg \varphi\})>\boldsymbol{I}(K)$, that is, supplementing $K$ with a tautology $\neg \varphi$ would increase the inconsistency degree: No inconsistency measure can be expected to do that. In other words, Proposition 1 is the most that can be obtained towards $\Vdash$ satisfying Reflexivity. The following example is an illustration of this.

Example 1. Consider the inconsistency measure $\boldsymbol{I}_{M I}$ satisfying Penalty, and an inconsistent knowledge base $K=\{a, \neg a, a \wedge \neg a\}$. Let $a$ be a consistent element of $K$. It is easy to see that $K \Vdash a$, since $2=\boldsymbol{I}_{M I}(K)>\boldsymbol{I}_{M I}(K \backslash\{\neg a\})=1$. However, for the inconsistent element $a \wedge \neg a$ of $K$, we have $K \forall a \wedge \neg a$ due to $\boldsymbol{I}_{M I}(K \cup\{\neg(a \wedge \neg a)\})=\boldsymbol{I}_{M I}(K)=2$.

We now turn to Monotony. To start with, Proposition 2 (proved by applying Lemma 2 twice) states that Monotony succeeds when $\psi=\neg \varphi$. A more substantial result is offered by Proposition 3 .

Proposition 2. If $K \Vdash \varphi$ then $K \cup\{\neg \varphi\} \Vdash \varphi$.

Proposition 3. Let I satisfy Free-formula Independence. Let $\psi$ be free for $K \cup$ $\{\neg \varphi\}$. If $K \Vdash \varphi$ then $K \cup\{\psi\} \Vdash \varphi$.

Proof. (i) First, consider the case $\neg \varphi \notin K$. Assume that $\psi$ is free for $K \cup\{\neg \varphi\}$. In view of Free-formula Independence, $\boldsymbol{I}(K \cup\{\neg \varphi\} \cup\{\psi\})=\boldsymbol{I}(K \cup\{\neg \varphi\})$. Also by Free-formula Independence, $\boldsymbol{I}(K)=\boldsymbol{I}(K \cup\{\psi\})$ because $\psi$ being free for $K \cup\{\neg \varphi\}$ entails $\psi$ being free for $K$. However, Lemma 1 gives $\boldsymbol{I}(K \cup\{\neg \varphi\})>$ $\boldsymbol{I}(K)$ due to $K \Vdash \varphi$ and $\neg \varphi \notin K$. Hence, $\boldsymbol{I}(K \cup\{\psi\} \cup\{\neg \varphi\})>\boldsymbol{I}(K \cup\{\psi\})$. Should $\psi=\neg \varphi$, a contradiction $\boldsymbol{I}(K \cup\{\psi\})>\boldsymbol{I}(K \cup\{\psi\})$ would arise. Hence, $\psi \neq \neg \varphi$.

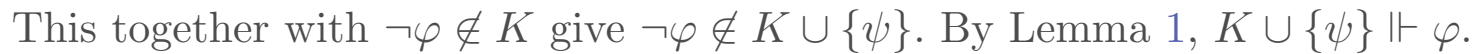

(ii) Second, consider the case $\neg \varphi \in K$. Then $K=K \cup\{\neg \varphi\}$. Also, $K \Vdash \varphi$ gives $K \backslash\{\neg \varphi\} \Vdash \varphi$ according to Lemma 2. Now, $\neg \varphi \notin K \backslash\{\neg \varphi\}$. Moreover, $\psi$ is free for $(K \backslash\{\neg \varphi\}) \cup\{\neg \varphi\}$ because $\psi$ is free for $K$. (i) can be applied, giving $(K \backslash\{\neg \varphi\}) \cup\{\psi\} \Vdash \varphi$. For $\psi \neq \neg \varphi$, Lemma 1 then gives $\boldsymbol{I}((K \backslash\{\neg \varphi\}) \cup\{\psi\} \cup$ $\{\neg \varphi\})>\boldsymbol{I}((K \backslash\{\neg \varphi\}) \cup\{\psi\})$. That is, $\boldsymbol{I}(K \cup\{\psi\} \cup\{\neg \varphi\})>\boldsymbol{I}((K \backslash\{\neg \varphi\}) \cup$ $\{\psi\})$. Therefore $\boldsymbol{I}(K \cup\{\psi\} \cup\{\neg \varphi\})>\boldsymbol{I}((K \cup\{\psi\}) \backslash\{\neg \varphi\})$ due to $\psi \neq \neg \varphi$. Per Definition 2, $K \cup\{\psi\} \Vdash \varphi$. The remaining case $\psi=\neg \varphi$ is obvious since $\neg \varphi \in K$.

Example 2. This example shows that Proposition 3 is the most that can be obtained towards $\Vdash$ satisfying Monotony. Consider the drastic measure $\boldsymbol{I}_{d}$ satisfying Free-Formula Independence. Let $K=\{a, \neg a\}, \varphi=a$, and $\psi=b \wedge \neg b$. 
Then $\psi$ is not free for $K \cup\{\neg \varphi\}$. Besides, we have $K \Vdash \varphi$. However, $K \cup\{\psi\} \forall \varphi$ since $\boldsymbol{I}_{d}(K \cup\{\psi\} \cup\{\neg \varphi\})=\boldsymbol{I}_{d}((K \cup\{\psi\}) \backslash\{\neg \varphi\})=1$.

Since Monotony seems to fail on a general count, we can look at a weakening known as Cautious Monotony [8]. To show under which conditions Cautious Monotony and Cut are satisfied, we propose, from a technical point of view, two postulates, Denial Elimination and Denial Preservation which are basically the converse of each other. Denial Preservation states that if a formula increases the inconsistency of a knowledge base, then for its superset with the same inconsistency, we may wish that the negation of the formula increases the same inconsistency to the knowledge base and the superset.

Cautious Monotony. If $K \Vdash \varphi$ and $K \Vdash \psi$ then $K \cup\{\psi\} \Vdash \varphi$.

Denial Elimination: Let $K$ and $\varphi$ be such that $\boldsymbol{I}(K \cup\{\varphi\})>\boldsymbol{I}(K)$. For all $K^{\prime} \supseteq K$, if $\boldsymbol{I}\left(K^{\prime} \cup\{\neg \varphi\}\right)=\boldsymbol{I}(K \cup\{\neg \varphi\})$ then $\boldsymbol{I}\left(K^{\prime}\right)=\boldsymbol{I}(K)$.

Denial Preservation: Let $K$ and $\varphi$ be such that $\boldsymbol{I}(K \cup\{\varphi\})>\boldsymbol{I}(K)$. For all $K^{\prime} \supseteq K$, if $\boldsymbol{I}\left(K^{\prime}\right)=\boldsymbol{I}(K)$ then $\boldsymbol{I}\left(K^{\prime} \cup\{\neg \varphi\}\right)=\boldsymbol{I}(K \cup\{\neg \varphi\})$.

Proposition 4. If I satisfies Denial Elimination, Monotony, and Rewriting, then $\Vdash$ satisfies Cautious Monotony restricted to the case $\neg \varphi \notin K$ and $\neg \psi \notin K$.

Proof. Let $K \Vdash \varphi$ and $K \Vdash \psi$. Assume $\neg \varphi \notin K$. Firstly, consider $\neg \varphi=\psi$. $K \Vdash \varphi$ gives $\boldsymbol{I}(K \cup\{\neg \varphi\})>\boldsymbol{I}(K)$ by Lemma 1. That is, $\boldsymbol{I}(K \cup\{\psi\} \cup\{\neg \varphi\})>$ $\boldsymbol{I}((K \cup\{\psi\}) \backslash\{\neg \varphi\})$. Hence, $K \cup\{\psi\} \Vdash \varphi$ by Definition 2. Secondly, consider $\neg \varphi \neq \psi$. Then $\neg \varphi \notin K \cup\{\psi\}$. Assume further $\neg \psi \notin K$. Applying Lemma 1 to $K \Vdash \psi$ yields $\boldsymbol{I}(K \cup\{\neg \psi\})>\boldsymbol{I}(K)$. Denial Elimination can be applied so that $\boldsymbol{I}(K \cup\{\neg \psi\}) \neq \boldsymbol{I}(K)$ entails $\boldsymbol{I}(K \cup\{\neg \varphi\} \cup\{\neg \neg \psi\}) \neq \boldsymbol{I}(K \cup\{\neg \neg \psi\})$. Then by Rewriting, we have $\boldsymbol{I}(K \cup\{\neg \varphi\} \cup\{\psi\}) \neq \boldsymbol{I}(K \cup\{\psi\})$. Due to Monotony, $\boldsymbol{I}(K \cup\{\neg \varphi\} \cup\{\psi\})>\boldsymbol{I}(K \cup\{\psi\})$. Eventually, $K \cup\{\psi\} \Vdash \varphi$ by Lemma 1.

Proposition 5. If I satisfies Denial Preservation, Monotony, and Rewriting, then $\Vdash$ satisfies Cut restricted to the case $\neg \varphi \notin K$ and $\neg \psi \notin K$.

Proof. The proof is similar to the converse of the proof of Proposition 4.

\section{$4 \quad$ KLM Properties Checking}

Kraus, Lehman and Magidor [7] introduced a series of possible properties for inference relations. In this section, we explore under which conditions the following KLM properties can be satisfied. More specifically, when an inconsistency measures satisfies some postulate(s), we show that the induced inference relation satisfies some KLM properties restricted to compulsory extra condition(s).

Right Weakening. If $K \Vdash \varphi$ and $\varphi \models \psi$ then $K \Vdash \psi$.

Left Logical Equivalence. If $K \equiv K^{\prime}$ and $K \Vdash \varphi$, then $K^{\prime} \Vdash \varphi$. 
AND. If $K \Vdash \varphi$ and $K \Vdash \psi$ then $K \Vdash \varphi \wedge \psi$.

Rational Monotony. If $K \Vdash \varphi$ and $K \Vdash \neg \psi$ then $K \cup\{\psi\} \Vdash \varphi$.

Contraposition. If $\varphi \Vdash \psi$ then $\neg \psi \Vdash \neg \varphi$.

OR. If $K \cup\{\varphi\} \Vdash \rho$ and $K \cup\{\psi\} \Vdash \rho$ then $K \cup\{\varphi \vee \psi\} \Vdash \rho$.

Below are the rationality postulates $[1,15]$ we will consider in this section. Note that Antinomy Increase and Mutual Increase are new rationality postulates. The former states that adding an inconsistent formula would increase the inconsistency degree. The latter states that when adding a formula $\varphi$ increases the inconsistency amount of an inconsistent knowledge base $K$, we would expect that adding $K$ also increases the inconsistency amount of $\varphi$.

Dominance: If $\varphi \notin K, \varphi \not \models \perp$ and $\varphi \models \psi$ then $\boldsymbol{I}(K \cup\{\varphi\}) \geq \boldsymbol{I}(K \cup\{\psi\})$.

Disjunct Minimality: $\min (\boldsymbol{I}(K \cup\{\varphi\}), \boldsymbol{I}(K \cup\{\psi\})) \leq \boldsymbol{I}(K \cup\{\varphi \vee \psi\})$.

Disjunct Maximality: $\boldsymbol{I}(K \cup\{\varphi \vee \psi\}) \leq \max (\boldsymbol{I}(K \cup\{\varphi\}), \boldsymbol{I}(K \cup\{\psi\}))$.

Antinomy Increase: Let $\models \neg \varphi$. If $\varphi \notin K$ then $\boldsymbol{I}(K \cup\{\varphi\})>\boldsymbol{I}(K)$.

Mutual Increase: If $K \models \perp$ and $\boldsymbol{I}(K \cup\{\varphi\})>\boldsymbol{I}(K)$, then $\boldsymbol{I}(K \cup\{\varphi\})>\boldsymbol{I}(\{\varphi\})$.

Proposition 6. Let I satisfy Dominance. Then $\Vdash$ satisfies Right Weakening restricted to the case $\neg \psi \forall \models \perp, \neg \psi \notin K$ and $\neg \varphi \notin K$.

Proof. Assume that $K \Vdash \varphi$ and $\varphi \models \psi$. Then $\neg \psi \models \neg \varphi$. By Dominance, we have $\boldsymbol{I}(K \cup\{\neg \psi\}) \geq \boldsymbol{I}(K \cup\{\neg \varphi\})$. We also have $\boldsymbol{I}(K \cup\{\neg \varphi\})>\boldsymbol{I}(K)$ by Lemma 1. Immediately, $\boldsymbol{I}(K \cup\{\neg \psi\})>\boldsymbol{I}(K)$. That is, $K \Vdash \psi$ by Lemma 1 .

Example 3. The extra condition $\neg \varphi \notin K$ of Proposition 6 is compulsory. Actually, consider $\boldsymbol{I}_{d}$ satisfying Dominance. Let $K=\{a, \neg a\}, \varphi=a$ and $\psi=a \vee b$. It follows that $K \Vdash \varphi$ and $\varphi \models \psi$. But $K \forall \psi$ since $\boldsymbol{I}_{d}(K \cup\{\neg \psi\})=\boldsymbol{I}_{d}(K)=1$.

Due to failure of the general form for Right Weakening, a special case is of interest: Is every tautology a consequence according to $\Vdash$ ?

Proposition 7. If I satisfies Antinomy Increase, $K \Vdash \varphi$ for every tautology $\varphi$.

Proof. Take a tautology $\varphi$. Thus, $\neg \neg \varphi$ is also a tautology. Assume $\neg \varphi \notin K$. By Antinomy Increase, $\boldsymbol{I}(K \cup\{\neg \varphi\})>\boldsymbol{I}(K)$. Hence, $K \Vdash \varphi$ by Lemma 1 . Assume $\neg \varphi \in K$. Then, $\neg \varphi \notin K \backslash\{\neg \varphi\}$. Therefore, $\boldsymbol{I}((K \backslash\{\neg \varphi\}) \cup\{\neg \varphi\})>\boldsymbol{I}(K \backslash\{\neg \varphi\})$ by Antinomy Increase. That is $\boldsymbol{I}(K)>\boldsymbol{I}(K \backslash\{\neg \varphi\})$. So $K \Vdash \varphi$ by Lemma 1 .

Using the previous proposition, we can get closer to Right Weakening.

Corollary 1. Let $\boldsymbol{I}$ satisfy Dominance and Antinomy Increase. Let $\neg \psi \notin K$ and $\neg \varphi \notin K$. If $K \Vdash \varphi$ and $\varphi \models \psi$ then $K \Vdash \psi$.

Proposition 8. Let $K \forall \neq \perp$. Then $\Vdash$ satisfies Left Logical Equivalence.

Proof. Apply Theorem 1 twice.

Example 4. The extra condition $K \forall \perp$ is necessary. Actually, consider $\boldsymbol{I}_{M I}$, and two inconsistent knowledge bases $K=\{a, \neg a\}$ and $K^{\prime}=\{b, \neg b\}$. It is obvious that $K \equiv K^{\prime}$ and $K \Vdash a$. However, $K^{\prime} \forall a$ as $\boldsymbol{I}\left(K^{\prime} \cup\{\neg a\}\right)=\boldsymbol{I}\left(K^{\prime}\right)=1$. 
Proposition 9. Let $\boldsymbol{I}$ satisfy Disjunct Minimality, Rewriting and Monotony. Then $\Vdash$ satisfies AND restricted to the case $\neg \varphi \notin K$ and $\neg \psi \notin K$.

Proof. Assume that $K \Vdash \varphi$ and $K \Vdash \psi$. Let $\neg \varphi \notin K$ and $\neg \psi \notin K$. Then $\boldsymbol{I}(K \cup\{\neg \varphi\})>\boldsymbol{I}(K)$ and $\boldsymbol{I}(K \cup\{\neg \psi\})>\boldsymbol{I}(K)$ by Lemma 1. Applying Disjunct Minimality gives $\boldsymbol{I}(K \cup\{\neg \varphi \vee \neg \psi\}) \geq \min (\boldsymbol{I}(K \cup\{\neg \varphi\}), \boldsymbol{I}(K \cup\{\neg \psi\}))$. Hence, $\boldsymbol{I}(K \cup\{\neg \varphi \vee \neg \psi\})>\boldsymbol{I}(K)$. Applying Rewriting yields $\boldsymbol{I}(K \cup\{\neg(\varphi \wedge \psi)\})>\boldsymbol{I}(K)$. Besides, applying Monotony gives $\boldsymbol{I}(K) \geq \boldsymbol{I}(K \backslash\{\neg(\varphi \wedge \psi)\})$. Therefore, it is the case that $\boldsymbol{I}(K \cup\{\neg(\varphi \wedge \psi)\})>\boldsymbol{I}(K \backslash\{\neg(\varphi \wedge \psi)\})$. By Definition $2, K \Vdash \varphi \wedge \psi$.

Example 5. Consider the drastic inconsistency measure $\boldsymbol{I}_{d}$ satisfying Disjunct Minimality, Rewriting and Monotony. Let $K=\{a, \neg a\}$ and $\varphi=\psi=a$. Then $\neg \psi$ and $\neg \varphi$ are formulas occurring in $K$. Besides, $K \Vdash \varphi$ and $K \Vdash \psi$. But we have $K \forall \varphi \wedge \psi$ due to $\boldsymbol{I}_{d}(K \cup\{\neg(\varphi \wedge \psi)\})=\boldsymbol{I}_{d}(K \backslash\{\neg(\varphi \wedge \psi)\})=1$.

The converse of the second item of Lemma 4 is untrue because $\boldsymbol{I}(K \cup\{\psi\})=$ $\boldsymbol{I}(K)$ is possible for $\psi$ not free for $K$ (see for instance the drastic inconsistency measure). However, by Monotony, it is possible to use $K \forall \neg \psi$ to infer $\boldsymbol{I}(K \cup$ $\{\psi\})=\boldsymbol{I}(K)$. That is the interest of the next lemma.

Lemma 5. Let I satisfy Monotony and Rewriting. Let $K \backslash\{\neg \varphi\} \Downarrow \neg \psi$. If $K \Vdash \varphi$ then $K \cup\{\psi\} \Vdash \varphi$.

Proof. First, consider $\neg \varphi \notin K$. The assumption becomes $K \forall \neg \psi$. Since the codomain of $\boldsymbol{I}$ is linearly ordered, Lemma 1 gives $\boldsymbol{I}(K \cup\{\neg \neg \psi\}) \leq \boldsymbol{I}(K)$. Using Rewriting, $\boldsymbol{I}(K \cup\{\psi\}) \leq \boldsymbol{I}(K)$. Applying Monotony gives $\boldsymbol{I}(K \cup\{\psi\})=\boldsymbol{I}(K)$. However, Lemma 1 gives $\boldsymbol{I}(K \cup\{\neg \varphi\})>\boldsymbol{I}(K)$ due to $K \Vdash \varphi$ and $\neg \varphi \notin K$. Therefore, $\boldsymbol{I}(K \cup\{\neg \varphi\})>\boldsymbol{I}(K \cup\{\psi\})$. By Monotony, $\boldsymbol{I}(K \cup\{\neg \varphi\} \cup\{\psi\}) \geq$ $\boldsymbol{I}(K \cup\{\neg \varphi\})$ hence $\boldsymbol{I}(K \cup\{\neg \varphi\} \cup\{\psi\})>\boldsymbol{I}(K \cup\{\psi\})$. Should $\psi=\neg \varphi$, a contradiction $\boldsymbol{I}(K \cup\{\psi\})>\boldsymbol{I}(K \cup\{\psi\})$ would arise. Hence, $\psi \neq \neg \varphi$. This together with $\neg \varphi \notin K$ give $\neg \varphi \notin K \cup\{\psi\}$. By Lemma 1, $K \cup\{\psi\} \Vdash \varphi$ ensues.

Consider now $\neg \varphi \in K$. Since $K \backslash\{\neg \varphi\} \| \forall \neg \psi$, in view of Lemma 1 , we have $\boldsymbol{I}((K \backslash\{\neg \varphi\}) \cup\{\neg \neg \psi\}) \leq \boldsymbol{I}(K \backslash\{\neg \varphi\})$. However, $\boldsymbol{I}(K \backslash\{\neg \varphi\})<\boldsymbol{I}(K)$ by Lemma 1 due to $K \Vdash \varphi$. Also, Monotony requires $\boldsymbol{I}(K) \leq \boldsymbol{I}(K \cup\{\psi\})$. Combining, $\boldsymbol{I}((K \backslash\{\neg \varphi\}) \cup\{\neg \neg \psi\})<\boldsymbol{I}(K \cup\{\psi\})$ ensues. By Rewriting, $\boldsymbol{I}((K \backslash\{\neg \varphi\}) \cup$ $\{\psi\})<\boldsymbol{I}(K \cup\{\psi\})$. Should $\psi=\neg \varphi$, a contradiction $\boldsymbol{I}(K \cup\{\psi\})<\boldsymbol{I}(K \cup\{\psi\})$ would arise. Hence, $\psi \neq \neg \varphi$. Thus, $\boldsymbol{I}((K \cup\{\psi\}) \backslash\{\neg \varphi\})<\boldsymbol{I}(K \cup\{\psi\})$. Applying Lemma 1, this gives $K \cup\{\psi\} \Vdash \varphi$.

Proposition 10. Let $\boldsymbol{I}$ satisfy Monotony and Rewriting. Then $\Vdash$ satisfies Rational Monotony restricted to the case $\neg \varphi \notin K$.

Proof. Apply Lemma 5 for $\neg \varphi \notin K$ in order to obtain that if $K \Vdash \varphi$ and $K \forall \neg \psi$ then $K \cup\{\psi\} \Vdash \varphi$.

Example 6. The extra condition $\neg \varphi \notin K$ in Proposition 10 is compulsory. Actually, consider $\boldsymbol{I}_{d}$ which satisfies Monotony and Rewriting. Let $K=\{a, \neg a, \neg b\}$, $\varphi=a$ and $\psi=b$. Trivially, $\neg \varphi \in K$. Also, $K \Vdash \varphi$ since $1=\boldsymbol{I}_{d}(K)>$ $\boldsymbol{I}_{d}(K \backslash\{\neg \varphi\})=0$. On the other hand, $K \forall \neg \psi$ since $\boldsymbol{I}_{d}(K \cup\{\neg \neg \psi\})=\boldsymbol{I}_{d}(K)=$ 1. However, $K \cup\{\psi\} \forall \varphi$ because $\boldsymbol{I}_{d}(K \cup\{\psi\})=\boldsymbol{I}_{d}((K \cup\{\psi\}) \backslash\{\neg \varphi\})=1$. 
Proposition 11. Let I satisfy Rewriting and Mutual Increase. Then $\Vdash$ satisfies Contraposition restricted to the case $\varphi \models \perp, \neg \psi \neq \varphi$ and $\psi \neq \neg \varphi$.

Proof. Assume $\varphi \Vdash \psi$. Then $\boldsymbol{I}(\{\varphi\} \cup\{\neg \psi\})>\boldsymbol{I}(\{\varphi\})$ by Lemma 1. Applying Mutual Increase to $\{\varphi\}$ and $\{\neg \psi\}$ yields $\boldsymbol{I}(\{\varphi\} \cup\{\neg \psi\})>\boldsymbol{I}(\{\neg \psi\})$. In view of Rewriting, $\boldsymbol{I}(\{\neg \neg \varphi\} \cup\{\neg \psi\})>\boldsymbol{I}(\{\neg \psi\})$. Hence, $\neg \psi \Vdash \neg \varphi$ by Lemma 1 .

Example 7. The extra condition $\neg \psi \neq \varphi$ is compulsory. Actually, consider $\boldsymbol{I}_{d}$ satisfying Rewriting and Mutual Increase. Let $\neg \psi=\varphi=\neg(a \vee \neg a)$. Then $\psi=a \vee \neg a . \varphi \Vdash \psi$ since $\left.\boldsymbol{I}_{d}(\{\varphi\} \cup\{\neg \psi\})=1>0=\boldsymbol{I}_{d}(\{\varphi\}) \backslash\{\neg \psi\}\right)$. But $\neg \psi \mid \forall \neg \varphi$ since $\boldsymbol{I}_{d}(\{\neg \psi\} \cup\{\neg \neg \varphi\})=\boldsymbol{I}_{d}(\{\neg \psi\} \backslash\{\neg \neg \varphi\})=1$.

A general result for OR is hard to obtain, since conditions satisfying OR are very demanding. Assessing inconsistency of a disjunction is a delicate matter. There is a lack of measures for assessing inconsistency of a disjunction in the existing literature except Disjunct Minimality and Disjunct Maximality [1]. However, even if an inconsistency measure satisfies these two postulates, it is not clear whether OR is satisfied by the induced inference relation.

Assume that $K \cup\{\varphi\} \Vdash \rho$ and $K \cup\{\psi\} \Vdash \rho$. Then by Definition 2, $\boldsymbol{I}(K \cup$ $\{\varphi\} \cup\{\neg \rho\})>\boldsymbol{I}((K \cup\{\varphi\}) \backslash\{\neg \rho\})$ and $\boldsymbol{I}(K \cup\{\psi\} \cup\{\neg \rho\})>\boldsymbol{I}((K \cup\{\psi\}) \backslash\{\neg \rho\})$. But $K \cup\{\varphi \vee \psi\} \Vdash \rho$ is not necessarily entailed by these conditions. Let us analyze this by considering two cases. Firstly, consider the case $\min (\boldsymbol{I}(K \cup\{\varphi\} \cup$ $\{\neg \rho\}), \boldsymbol{I}(K \cup\{\psi\} \cup\{\neg \rho\}))=\boldsymbol{I}(K \cup\{\varphi\} \cup\{\neg \rho\})$. Then $\boldsymbol{I}(K \cup\{\varphi \vee \psi\} \cup\{\neg \rho\}) \geq$ $\boldsymbol{I}(K \cup\{\varphi\} \cup\{\neg \rho\})$ by Disjunct Minimality. In such a case, we are not able to compare $\boldsymbol{I}(K \cup\{\varphi \vee \psi\} \cup\{\rho\})$ and $\boldsymbol{I}((K \cup\{\varphi \vee \psi\}) \backslash\{\rho\})$ since the relative order between $\boldsymbol{I}((K \cup\{\varphi\}) \backslash\{\neg \rho\})$ and $\boldsymbol{I}((K \cup\{\varphi\}) \backslash\{\neg \rho\})$ is not clear. Both $\boldsymbol{I}((K \cup$ $\{\varphi\}) \backslash\{\neg \rho\}) \leq \boldsymbol{I}((K \cup\{\varphi\}) \backslash\{\neg \rho\})$ and $\boldsymbol{I}((K \cup\{\varphi\}) \backslash\{\neg \rho\}) \geq \boldsymbol{I}((K \cup\{\varphi\}) \backslash\{\neg \rho\})$ are possible. If $\boldsymbol{I}((K \cup\{\varphi\}) \backslash\{\neg \rho\}) \leq \boldsymbol{I}((K \cup\{\varphi\}) \backslash\{\neg \rho\})$, we have no idea which one is smaller.

However, it is possible to compare the two items when $\boldsymbol{I}((K \cup\{\varphi\}) \backslash\{\neg \rho\}) \geq$ $\boldsymbol{I}((K \cup\{\varphi\}) \backslash\{\neg \rho\})$. Let us consider a simple case where $\neg \rho \neq \varphi, \neg \rho \neq \psi$ and $\neg \rho \neq \varphi \vee \psi$. Then $\boldsymbol{I}((K \cup\{\varphi\}) \backslash\{\neg \rho\}) \geq \boldsymbol{I}((K \cup\{\varphi\}) \backslash\{\neg \rho\})$ is equivalent to $\boldsymbol{I}((K \backslash\{\neg \rho\}) \cup\{\varphi\}) \geq \boldsymbol{I}((K \backslash\{\neg \rho\}) \cup\{\psi\})$. Applying Disjunct Maximality gives $\boldsymbol{I}((K \backslash\{\neg \rho\}) \cup\{\varphi\}) \geq \boldsymbol{I}((K \backslash\{\neg \rho\}) \cup\{\varphi \vee \psi\})$, namely, $\boldsymbol{I}((K \cup\{\varphi\}) \backslash\{\neg \rho\}) \geq$ $\boldsymbol{I}((K \cup\{\varphi \vee \psi\}) \backslash\{\neg \rho\})$. Thus, $\boldsymbol{I}(K \cup\{\varphi \vee \psi\} \backslash\{\neg \rho\})>\boldsymbol{I}((K \cup\{\varphi \vee \psi\}) \backslash\{\neg \rho\})$. By Definition 2, $K \cup\{\varphi \vee \psi\} \Vdash \rho$. The analysis for the case where $\min (\boldsymbol{I}(K \cup$ $\{\varphi\} \cup\{\neg \rho\}), \boldsymbol{I}(K \cup\{\psi\} \cup\{\neg \rho\}))=\boldsymbol{I}(K \cup\{\psi\} \cup\{\neg \rho\})$ is similar to the first case.

\section{$5 \quad$ Paraconsistency Checking}

This section investigates which inconsistency measures induce a paraconsistent inference relation. The following definition formally describes paraconsistency.

Definition 3 (Paraconsistency). An inference relation $\Vdash$ is paraconsistent if for all inconsistent $K \in \mathcal{K}$, there exists a formula $\varphi$ such that $K \forall \varphi$. 
An inconsistency measure $\boldsymbol{I}$ is called relative [2] if it satisfies Normalization (i.e., $0 \leq \boldsymbol{I}(K) \leq 1$ ) and either Free-Formula Reduction or Relative Separability (or both).

Free-Formula Reduction: For $\varphi \notin K$, if $\varphi$ is free for $K$ and $\boldsymbol{I}(K) \neq 0$ then $\boldsymbol{I}(K \cup\{\varphi\})<\boldsymbol{I}(K)$.

Relative Separability: If $\boldsymbol{I}\left(K_{1}\right) \lesssim \boldsymbol{I}\left(K_{2}\right)$ and $\operatorname{Atoms}\left(K_{1}\right) \cap \operatorname{Atoms}\left(K_{2}\right)=\varnothing$ then $\boldsymbol{I}\left(K_{1}\right) \lesssim \boldsymbol{I}\left(K_{1} \cup K_{2}\right) \lesssim \boldsymbol{I}\left(K_{2}\right)$ where either $\lesssim$ is $<$ in every instance or $\lesssim$ is $=$ in every instance.

Proposition 12. If $\boldsymbol{I}$ is an inconsistency measure satisfying (i) Free-Formula Reduction or (ii) Relative Separability, then $\Vdash$ is paraconsistent.

Proof. (i) Take an inconsistent $K \in \mathcal{K}$ and an atom $a \notin \operatorname{Atoms}(K)$. Then $\neg a$ is free for $K$ and $\neg a \notin K$. Besides, $\boldsymbol{I}(K) \neq 0$ by Consistency Null. So applying FreeFormula Reduction to $K$ gives $\boldsymbol{I}(K \cup\{\neg a\})<\boldsymbol{I}(K)$. Thus, $K \| \varphi$ by Lemma 1 . Hence, $\Vdash$ is paraconsistent by Definition 3. (ii) Take an inconsistent $K \in \mathcal{K}$ and an atom $a \notin \operatorname{Atoms}(K)$. Then $\boldsymbol{I}(K)>0$ and $\boldsymbol{I}(\{\neg a\})=0$ by Consistency Null. Hence, we have $\boldsymbol{I}(\{\neg a\})<\boldsymbol{I}(K)$. Besides, Atoms $(\{\neg a\}) \cap \operatorname{Atoms}(K)=\varnothing$. Applying Relative Separability gives $\boldsymbol{I}(\{\neg a\})<\boldsymbol{I}(K \cup\{\neg a\})<\boldsymbol{I}(K)$. Therefore, $K \Downarrow a$ by Lemma 1 . Thus, $\Vdash$ is paraconsistent by Definition 3 .

Theorem 2. For every relative inconsistency measure $\boldsymbol{I}$, the inference relation $\Vdash$ induced from $\boldsymbol{I}$ is paraconsistent.

Next we show that the inference relation $\Vdash$ induced from $\boldsymbol{I}$ is paraconsistent if $\boldsymbol{I}$ satisfies some rationality postulate(s). Note that we propose a postulate Tautology Non-Increase which states that any tautology does not increase inconsistency degree.

Safe-Formula Independence: If $\varphi$ is safe for $K$ then $\boldsymbol{I}(K \cup\{\varphi\})=\boldsymbol{I}(K)$.

Tautology Independence: If $\varphi$ is a tautology then $\boldsymbol{I}(K \cup\{\varphi\})=\boldsymbol{I}(K)$.

MI-separability: If $\mathrm{MI}\left(K_{1} \cup K_{2}\right)=\operatorname{MI}\left(K_{1}\right) \cup \operatorname{MI}\left(K_{2}\right)$ and $\operatorname{MI}\left(K_{1}\right) \cap \mathrm{MI}\left(K_{2}\right)=\varnothing$ then $\boldsymbol{I}\left(K_{1} \cup K_{2}\right)=\boldsymbol{I}\left(K_{1}\right)+\boldsymbol{I}\left(K_{2}\right)$.

Tautology Non-Increase: If $\varphi$ is a tautology then $\boldsymbol{I}(K \cup\{\varphi\}) \leq \boldsymbol{I}(K)$.

Proposition 13. If $\boldsymbol{I}$ is an inconsistency measure satisfying (i) Free-formula Independence, (ii) Safe-Formula Independence, (iii) Tautology Independence or (iv) MI-separability, then $\Vdash$ is paraconsistent.

Proof. (i) Take an inconsistent $K \in \mathcal{K}$ and an atom $a \notin \operatorname{Atoms}(K)$. Then applying Free-Formula Independence to $K$ gives $\boldsymbol{I}(K \cup\{\neg a\})=\boldsymbol{I}(K)$, as $\neg a$ is free for $K$. Hence, $K \forall \varphi$ by Lemma 1. (ii) Obvious. (iii) Take an inconsistent $K \in \mathcal{K}$, and an inconsistent formula $\varphi$ such that no atom in $\varphi$ occur in $K$. Then applying Tautology Independence gives $\boldsymbol{I}(K \cup\{\neg \varphi\})=\boldsymbol{I}(K)$, as $\neg \varphi$ is a tautology. Trivially, $\neg \varphi \notin K$. Hence $K \forall \varphi$ by Lemma 1. (iv) Take an inconsistent $K \in \mathcal{K}$ and an atom $a \notin \operatorname{Atoms}(K)$. Then $\operatorname{MI}(K \cup\{\neg a\})=\operatorname{MI}(K)$ since $\neg a$ does not participate in inconsistency. Besides, we have $\operatorname{MI}(\{\neg a\})=\varnothing$. Hence, we have 
$\operatorname{MI}(K \cup\{\neg a\})=\operatorname{MI}(K) \cup \operatorname{MI}(\{\neg a\})$ and $\operatorname{MI}(K) \cap \operatorname{MI}(\{\neg a\})=\varnothing$. Therefore, $\boldsymbol{I}(K \cup\{\neg a\})=\boldsymbol{I}(K)+\boldsymbol{I}(\{\neg a\})$ holds by applying Ml-separability to $K$ and $\{\neg a\}$. Thus, $\boldsymbol{I}(K \cup\{\neg a\})=\boldsymbol{I}(K)$ due to $\boldsymbol{I}(\{\neg a\})=0$. So $K \forall a$ by Lemma 1 .

Lemma 6. If I satisfies Tautology Non-Increase, $K \Downarrow \varphi$ for all inconsistent $\varphi$.

Proof. Let $\varphi$ is be an inconsistent formula. Then $\neg \varphi$ is a tautology. Assume $\neg \varphi \notin$ $K$. According to Tautology Non-Increase, $\boldsymbol{I}(K \cup\{\varphi\}) \leq \boldsymbol{I}(K)$. Using Lemma 1 , $K \Downarrow \varphi$. Otherwise, assume $\neg \varphi \in K$. Applying Tautology Non-Increase, we have $\boldsymbol{I}((K \backslash\{\neg \varphi\}) \cup\{\neg \varphi\}) \leq \boldsymbol{I}(K \backslash\{\neg \varphi\})$. By Lemma $1, K \Downarrow \varphi$.

It is easy to see from Lemma 6 that if $\boldsymbol{I}$ satisfies Tautology Non-Increase then $\Vdash$ is paraconsistent.

\section{Dependencies and Incompatibilities}

Some postulates imply some other postulates, whereas others are incompatible. This section presents results in this sense.

Lemma 7. The following statements hold: (i) MI-separability entails Antinomy Increase and Tautology Independence. (ii) Penalty entails Antinomy Increase. (iii) Tautology Independence entails Tautology Non-Increase. (iv) Penalty and Monotony entails Mutual Increase.

Proof. (i) We first show that MI-separability entails Antinomy Increase. Let $\varphi \notin K$ such that $\varphi \models \perp$. Then, $\operatorname{MI}(K \cup\{\varphi\})=\operatorname{MI}(K) \cup \operatorname{MI}(\{\varphi\})$ and $\operatorname{MI}(K) \cap \operatorname{MI}(\{\varphi\})=\varnothing$. By Ml-separability, $\boldsymbol{I}(K \cup\{\varphi\})=\boldsymbol{I}(K)+\boldsymbol{I}(\{\varphi\})$. However, Consistency Null guarantees $\boldsymbol{I}(\{\varphi\})>0$. Then, $\boldsymbol{I}(K \cup\{\varphi\})>\boldsymbol{I}(K)$. We turn to show that Ml-separability entails Tautology Independence. Let $\varphi$ be a tautology. Then, $\operatorname{MI}(K \cup\{\varphi\})=\operatorname{MI}(K) \cup \operatorname{MI}(\{\varphi\})$ and $\operatorname{MI}(K) \cap \operatorname{MI}(\{\varphi\})=\varnothing$. Ml-separability then gives $\boldsymbol{I}(K \cup\{\varphi\})=\boldsymbol{I}(K)+\boldsymbol{I}(\{\varphi\})$. Consistency Null ensures $\boldsymbol{I}(\{\varphi\})=0$. Thus, $\boldsymbol{I}(K \cup\{\varphi\})=\boldsymbol{I}(K)$. (ii) Consider $\varphi \notin K$ such that $\varphi \models \perp$. Clearly, $\varphi$ is not free for $K$ because the empty set is a consistent subset $K$ but $\varnothing \cup\{\varphi\} \models \perp$. Applying Penalty, $\boldsymbol{I}(K \cup\{\varphi\})>\boldsymbol{I}(K)$. (iii) Obvious. (iv) Assume $K \models \perp$ and $\boldsymbol{I}(K \cup\{\varphi\})>\boldsymbol{I}(K)$. Then the latter assumption gives $\varphi \notin K$. Let us take a formula $\psi$ that belongs to a minimal inconsistent subset of $K$. Hence, $\psi$ is not free for $(K \backslash\{\psi\}) \cup\{\varphi\}$. Applying Penalty yields $\boldsymbol{I}(K \cup\{\varphi\})>\boldsymbol{I}((K \backslash\{\psi\}) \cup\{\varphi\})$. In view of Monotony, $\boldsymbol{I}((K \backslash\{\psi\}) \cup\{\varphi\}) \geq \boldsymbol{I}(\{\varphi\})$. Immediately, $\boldsymbol{I}(K \cup\{\varphi\})>\boldsymbol{I}(\{\varphi\})$.

Accordingly, if $\boldsymbol{I}$ satisfies Penalty then $K \Vdash \varphi$ for all consistent $\varphi \in K$ (by Proposition 1) and all tautological $\varphi$ (by Proposition 7).

The results given in the previous sections do not generally add up in the sense that combining rationality postulates for a given inconsistency measure may make the induced inference inference fails to enjoy some property. There are two main reasons. First, some possible properties of the induced inference relation are exclusive of each other. Second, some rationality postulates are incompatible. For 
Table 1. Satisfiable Properties of the Inference Relation $\Vdash$, depending on Sufficient Postulates of the Inconsistency Measure $\boldsymbol{I}$ and Compulsory Extra Conditions, with Reference to the Result

\begin{tabular}{l|l|l|l}
\hline Properties of $\Vdash$ & $\begin{array}{l}\text { Sufficient postulates of } \\
\boldsymbol{I}\end{array}$ & Extra conditions & Results \\
\hline Reflexivity & Penalty & Consistent formulas & Proposition 1 \\
\hline Monotony & $\begin{array}{l}\text { Free-formula } \\
\text { independence }\end{array}$ & $\psi$ is free for $K \cup\{\neg \varphi\}$ & Proposition 3 \\
\hline Cut & $\begin{array}{l}\text { Denial preservation, } \\
\text { monotony, rewriting }\end{array}$ & $\neg \varphi \notin K, \neg \psi \notin K$ & Proposition 5 \\
\hline Cautious monotony & $\begin{array}{l}\text { Denial elimination, } \\
\text { monotony, rewriting }\end{array}$ & $\neg \varphi \notin K, \neg \psi \notin K$ & Proposition 4 \\
\hline Right weakening & $\begin{array}{l}\text { Dominance, antinomy } \\
\text { increase }\end{array}$ & $\neg \varphi \notin K, \neg \psi \notin K$ & Corrolary 1 \\
\hline AND & $\begin{array}{l}\text { Disjunct minimality, } \\
\text { rewriting, monotony }\end{array}$ & $\neg \varphi \notin K, \neg \psi \notin K$ & Proposition 9 \\
\hline Rational monotony & $\begin{array}{l}\text { Monotony, rewriting } \\
\text { Rewriting, mutual } \\
\text { increase }\end{array}$ & $\begin{array}{l}\neg \notin \notin K \\
\text { Contraposition }\end{array}$ & $\begin{array}{l}\varphi \psi \neq \\
\text { Proposition 10 }\end{array}$ \\
\hline
\end{tabular}

instance, a consequence of Lemma 6 is that Tautology Non-Increase makes AND to fail whenever Reflexivity holds for all consistent formulas (let $K \supseteq\{\varphi, \neg \varphi\}$ for some consistent non-tautological $\varphi$ ). Then we have the following proposition.

Proposition 14. If I satisfies Penalty and Tautology Non-Increase, then $\Vdash$ fails to satisfy AND.

\section{Conclusion and Future Work}

In order to reason with inconsistent knowledge bases, we have proposed a new family of inference relations induced from inconsistency measures. It is worth mentioning that the new inference relations have the same consequences as classical logic when premises are consistent. We have shown that these inference relations satisfy some important nonmonotonic reasoning properties (restricted to some extra requirements) when the corresponding inconsistency measures satisfy some rationality postulates (see Table 1). Furthermore, we have shown that the new inference relations are paraconsistent when the corresponding inconsistency measures satisfy some rationality postulates; importantly, every relative inconsistency measures can induce a paraconsistent inference relation. Lastly, we have presented some dependencies and incompatibilities among postulates.

This article opens up directions for future research. Firstly, we will continue to investigate whether the remaining KLM properties (i.e. Equivalence, MPC, 
Transitivity, EHD, Loop) can be satisfied. We will also keep on investigating dependencies and incompatibilities between postulates. Secondly, we will consider an extension of the approach to infinite knowledge bases. An idea in this sense would be that the inference relation $\Vdash$ induced from an inconsistency measure $\boldsymbol{I}$, considering an infinite knowledge base $X$, would be such that: $X \Vdash \varphi$ iff $\boldsymbol{I}(Y \cup\{\neg \varphi\})>\boldsymbol{I}(Y \backslash\{\neg \varphi\})$ for some finite $Y \subseteq X$.

\section{References}

1. Besnard, P.: Revisiting postulates for inconsistency measures. In: Fermé, E., Leite, J. (eds.) JELIA 2014. LNCS (LNAI), vol. 8761, pp. 383-396. Springer, Cham (2014). https://doi.org/10.1007/978-3-319-11558-0_27

2. Besnard, P., Grant, J.: Relative inconsistency measures. Artif. Intell. 280, 103231 (2020)

3. Brown, B.: Inconsistency measures and paraconsistent consequence. In: Grant, J., Martinez, M.V. (eds.) Measuring Inconsistency in Information, Studies in Logic, vol. 73, pp. 219-233. College Publications (2018)

4. Grant, J., Hunter, A.: Measuring consistency gain and information loss in stepwise inconsistency resolution. In: Liu, W. (ed.) ECSQARU 2011. LNCS (LNAI), vol. 6717, pp. 362-373. Springer, Heidelberg (2011). https://doi.org/10.1007/978-3642-22152-1_31

5. Hunter, A., Konieczny, S.: On the measure of conflicts: shapley inconsistency values. Artif. Intell. 174(14), 1007-1026 (2010)

6. Konieczny, S., Lang, J., Marquis, P.: Quantifying information and contradiction in propositional logic through test actions. In: Gottlob, G., Walsh, T. (eds.) Proceedings of the 18th International Joint Conference on Artificial Intelligence (IJCAI 2003), 9-15 August , 2003, Acapulco, Mexico, pp. 106-111. Morgan Kaufmann (2003)

7. Kraus, S., Lehmann, D., Magidor, M.: Nonmonotonic reasoning, preferential models and cumulative logics. Artif. Intell. 44(1-2), 167-207 (1990)

8. Makinson, D.: General patterns in nonmonotonic reasoning. In: Gabbay, D.M., Hogger, C.J., Robinson, J.A. (eds.) Handbook of Logic in Artificial Intelligence and Logic Programming, vol. 3, pp. 35-110. Clarendon Press, Oxford (1994)

9. Mu, K., Liu, W., Jin, Z.: A general framework for measuring inconsistency through minimal inconsistent sets. J. Knowl. Inf. Syst. 27(1), 85-114 (2011)

10. Oller, C.: Measuring coherence using LP-models. J. Appl. Logic 2(4), 451-455 (2004)

11. Priest, G.: The logic of paradox. J. Philos. Logic 8(1), 219-241 (1979)

12. Scott, D.: Completeness and axiomatizability in many-valued logic. In: Henkin, L.A. (ed.) Tarski Symposium, Proceedings of Symposia in Pure Mathematics, Providence, RI, USA, vol. 25, pp. 411-436. American Mathematical Society (1974)

13. Tarski, A.: On some Fundamental Concepts of Metamathematics. In: Logic, Semantics, Metamathematics, 2nd edn, pp. 30-37. Hackett, Indianapolis (1983). Translated by J. H. Woodger, edited by John Corcoran

14. Thimm, M.: Measuring inconsistency in probabilistic knowledge bases. In: Bilmes, J.A., Ng, A.Y. (eds.) 25th Conference on Uncertainty in Artificial Intelligence (UAI 2009), 18-21 June 2009, Montreal, QC, Canada, pp. 530-537. AUAI Press (2009)

15. Thimm, M.: On the evaluation of inconsistency measures. In: Grant, J., Martinez, M.V. (eds.) Measuring Inconsistency in Information, Studies in Logic, vol. 73, pp. 19-60. College Publications (2018) 